REFERENCES:

[1] Henaux $S$ et al. Risk of losing remission, low disease activity or radiographic progression in case of bDMARD discontinuation or tapering in rheumatoid arthritis: systematic analysis of the literature and meta-analysis. Ann Rheum Dis 2018;77:515-522.

Disclosure of Interests: None declared

DOI: 10.1136/annrheumdis-2021-eular.3045

\section{AB0231 OUTCOMES IN RHEUMATOID ARTHRITIS PATIENTS UNDER TOCILIZUMAB AS FIRST BDMARD: A REAL- LIFE MONOCENTRIC COHORT STUDY}

F. R. Martins ${ }^{1}$, A. Martins ${ }^{2}$, D. Santos Oliveira ${ }^{2,3}$, D. Fonseca ${ }^{4}$, M. Rato ${ }^{2}$, F. Oliveira Pinheiro' ${ }^{2}$, S. Garcia ${ }^{2}$, B. M. Fernandes ${ }^{2}$, L. Costa ${ }^{2}$, M. Bernardes ${ }^{2}$. ${ }^{1}$ Centro Hospitalar Universitário do Algarve - Faro, Rheumathology, Faro, Portugal; ${ }^{2}$ Centro Hospitalar Universitário São João, Rheumathology, Porto, Portugal; ${ }^{3}$ Faculty of Medicine, University of Porto, Center for Health Technology and Services Research (CINTESIS), Porto, Portugal; ${ }^{4}$ C. H. Vila Nova de Gaial Espinho, Rheumathology, Vila Nova de Gaia, Portugal

Background: Rheumatoid arthritis (RA) is one of the most frequent systemic inflammatory rheumatic diseases, being constantly assessed regarding new disease activity monitoring tools and new therapeutic targets and therapies. Tocilizumab (TCZ) is one of the latest biological disease-modifying antirheumatic drugs (bDMARDs) approved for RA's treatment, usually as a second line agent in daily clinical practice.

Objectives: Evaluate the different disease and patient reported outcomes in patients undergoing treatment with tocilizumab as the first biologic therapy.

Methods: All patients with a definite RA diagnosis who had undergone treatment with TCZ as the first biologic therapy at a tertiary hospital's rheumatology department were included in this analysis. Diverse socio-demographic data, as well as disease and patient related outcomes were assessed at baseline, 6 and 12 months of treatment with TCZ, and posteriorly extracted from the Portuguese register of rheumatic diseases (Reuma.PT). Statistical analysis included non-parametric tests such as Wilcoxon test and univariate analysis using linear and logistic regression models.

Results: Fifty-one patients were included, $88.2 \%$ females, with a median age at introduction of TCZ of $53.5+/-10.4$ years; mainly seropositive for either rheumatoid factor $(66 \%)$ or anti citrullinated peptide antibody (ACPA; $68 \%$ ), with an erosive disease $(75.6 \%)$ and concomitantly treated with a conventional synthetic disease modifying anti-rheumatic drug (csDMARD) (70.5\%). During follow-up there was a statistically significant reduction at 6 and 12 months of TCZ treatment regarding DAS28 (4 variables) (4v) and DAS28(4V)-CRP scores ( $p<$ $0.001)$, SDAI $(p<0.001)$, CDAl $(p<0.001), 68 / 66$ tender and swollen joint counts (TJC/SJC) $(p<0.001)$, ESR and CRP $(p<0.001)$, patient and physician VAS $(p<0.001)$ and HAQ score $(p=0.01$ at 6 months and $p<0.001$ at 12 months). Rheumatoid factor and ACPA serum levels weren't statistically different at 6 and 12 months of treatment with TCZ compared to the initial assessment, as well as the ACR responders at the same 6 months versus those at 12 months. A majority of patients showed good EULAR response at $6(52.6 \%)$ and $12(56.3 \%)$ months, as well as moderate to high mean improvement in ACR core set measures at 6 $(53.3 \pm 22.7)$ and $12(54.3 \pm 25.2)$ months. Assessment of subsequent therapeutic maintenance showed that $75 \%$ of patients remained under tocilizumab with an average treatment duration of $48.8 \pm 37.7$ months. Reasons for switch ranged from adverse effects $(63.6 \%)$ to primary failure $(18.2 \%)$ and secondary failure $(18.2 \%)$. There was a significant reduction in DAS28(4V), DAS28(4V)-CRP, CDAl, SDAI, TJC and SJC, ESR, CRP, patient and physician VAS and HAQ scores between 6 and 12 months of therapy $(p<0,001)$. ACR and EULAR responses didn't differ significantly between assessments at 6 and 12 months. In the absence of a representative number of RA patients on TCZ monotherapy, it wasn't possible to draw conclusions about the need to use combined therapy with a csDMARD for better clinically significant response.

A higher degree of ACR response at 6 months was associated with higher serum rheumatoid factor levels (OR 1.13, $p<0.05)$ at baseline, while a lower degree of response was seen with higher TJC $(p=0.05)$ and HAQ score $(p<0.01)$. ACR response at 12 months was lower in patients with erosive disease at baseline $(p$ $<0.05$ ). Regarding EULAR response criteria at 6 months, there was a negative association with higher TJC $(p<0.05)$, while at 12 months the negative trend was associated with ESR levels $(p<0.05)$ and HAQ scores $(p<0.05)$ at baseline. Conclusion: There seems to be evidence of good therapeutic response to TCZ in bDMARD naïve RA patients assessed at 6 months from baseline, without evidence of significant improvement of response measures further down the line. Basal serum rheumatoid factor levels, TJC, HAQ scores and the presence of erosive disease may have some predictive value on the therapeutic response. Further studies comparing TCZ as the first bDMARD in naïve RA patients against TNF inhibitors are needed.

Disclosure of Interests: None declared

DOI: 10.1136/annrheumdis-2021-eular.3110

\section{AB0232 PREVALENCE OF INFECTIONS UNDER BIOTHERAPY} DURING RHEUMATOID ARTHRITIS

K. Baccouche ${ }^{1}$, R. Grassa ${ }^{1}$, S. Lataoui ${ }^{1}$, O. Jomaa ${ }^{1}$, N. El Amri ${ }^{1}$, H. Zeglaoui ${ }^{1}$, E. Bouajina ${ }^{1} .{ }^{1}$ Farhat Hached Hospital, Rheumathology, Sousse, Tunisia

Background: Biologic therapies are emerging as a significant therapeutic option for many with debilitating inflammatory and autoimmune conditions including rheumatoid arthritis (RA). These biological agents are highly effective in RA. Potential complications are dominated by infections.

Objectives: To evaluate the different infections occurred under biotherapy. Methods: This is a descriptive retrospective study including patients under biotherapy, hospitalized between 2000-2016 in the Rheumatology Department of Farhat Hached hospital in Sousse,Tunisia. We evaluated for each patient the different infections that have occurred, specifying the different types of biotherapy received.

Results: Sixty one patients are included ( 54 women and 7 men).The average age is 55.33 years \pm 11.51 [34-81 years]. We found 47 infections in 40 patients $(65.57 \%)$ with at least one infection under biotherapy: 41 women and 6 men with a mean age at 57.72 years [34-81 years]. Infections occurred under anti IL6 in $46.32 \%$, Infliximab in $31.58 \%$, anti CD20 in $11.58 \%$, Etanercept in $7.37 \%$ and under Certolizumab in $3.16 \%$. The infection was bacterial in $68.42 \%: 28$ pulmonary infections, 8 oto rhino laryngology infections, 14 urinary infections, 2 sof tissue abscess, 13 cutaneous infections, 02 ocular infections. A viral origin of the infection was noted in $12.63 \%$ of cases. As for mycosis infections they represent $18.75 \%$ of the etiologies identified. The treatment was medical in $100 \%$ of cases and the evolution was favorable in all patients without resorting to the definitive discontinuation of biotherapy.

Conclusion: The infections reported in our series are much more frequent under anti IL6 treatment and bacteria are the most incriminating pathogenic agents in our patients.

\section{REFERENCES:}

[1] Simon, T. A., and al. Comparative risk of malignancies and infections in patients with rheumatoid arthritis initiating abatacept versus other biologics: a multi-database real-world study. Arthritis Research \& Therapy, 21(1) (2019).

[2] Pawar, A.,and al Risk of serious infections in tocilizumab versus other biologic drugs in patients with rheumatoid arthritis: a multidatabase cohort study. Annals of the Rheumatic Diseases, annrheumdis-2018-214367(2019).

Disclosure of Interests: None declared

DOI: 10.1136/annrheumdis-2021-eular.3197

\section{$\mathrm{AB} 0233$ \\ REASONS AND RISK FACTOR FOR DISCONTINUATION OF BIOLOGIC AGENTS FOR RHEUMATOID ARTHRITIS PATIENTS IN LONG-TERM OBSERVATION}

K. Terabe ${ }^{1}$, N. Takahashi ${ }^{1}$, S. Asai ${ }^{1}$, Y. Hirano ${ }^{2}$, Y. Kanayama ${ }^{3}$, T. Kojima ${ }^{1}$.

${ }^{1}$ Nagoya University School of Medicie, Orthopedic Surgery, Nagoya, Japan;

${ }^{2}$ Toyohashi Municipal Hospital, Rheumatology, Toyohashi, Japan; ${ }^{3}$ Toyota Kosei

Hospital, Orthopedic Surgery, Toyota, Japan

Background: Rheumatoid arthritis (RA) patients who failed a first biologic agent due to any reasons have the option of switching to a second one along with the strategy of biologic agent treatment. Patients go over switching to the next one at failing their biologic agent. On the other hand, there are some patients who discontinue any biologic agent treatment due to various reasons such as tolerability concern, complications, economic issue, remission and so $\mathrm{on}^{12}$. The impact of this concern has been less studied.

Objectives: The objective of this study was to investigate the reasons and the risk factors for discontinuation any biologic agent in RA patients.

Methods: To Include patients who are observed long-term, patients who underwent biologic agent treatment between 2003 and 2007 at Nagoya University Hospital and 12 other institutes (Tsurumai Biologics Communication Study Group) were enrolled. 570 patients who were confirmed continuation or discontinuation of biologic agent treatment were enrolled. The last observation was September 2017. We analyzed the retention rate of biologic agent treatment and the reasons for discontinuation. To identify the risks for discontinuation, baseline demographics were compared between the continuing group and the disc continuing group using cox hazard regression analysis.

Results: In total 570 patients, the average duration of treatment with biologics was $6.6 \pm 3.3$ (years) and total patient-year was 3739 in this study. 458 patients were administered biologics continuously, 112 patients were withdrawn. Table 1 showed the demographic data in total patients. The retention rate was $96.0 \%$ (discontinuation $n=23$ ) at least 1 year from starting biologics treatment, $92.6 \%$ $(n=42)$ at 3 years, $88.2 \%(n=67)$ at 5 years, $84.4 \%(n=89)$ at 7 years, $81.1 \%$ $(n=108)$ at 10 years. In 112 patients who discontinued, the reasons were adverse events in 74 patients, lack of effectiveness in 11 patients, others in 27 patients. Comparison of incidence for discontinuation using cumulative hazard function, the reason of adverse events was significantly higher than others reasons 
(Figure 1). To identify the risks of discontinuation, we analyzed by multivariable Cox proportional hazard modeling in patients who discontinued treatment due to adverse events, the risk factors (hazard ratio: $\mathrm{HR}$, confidence interval: $\mathrm{Cl}$ ) were over 3 of Steinblocker class (HR 1.85 [1.02-2.04]), age (HR:1.07 [1.04-1.10]) and Non-concomitant with methotrexate (HR 1.90 [1.08-3.33]) (Figure 2).

Table 1.

\begin{tabular}{lc}
\hline Age (years) & $56.1 \pm 13.4$ \\
Gender & \\
$\mathrm{n}(\%$ male) & $110(19 \%)$ \\
$\mathrm{n}(\%$ female) & $460(81 \%)$ \\
Disease duration (years) & $11.1 \pm 9.8$ \\
stage & \\
1,2 & $104(19 \%)$ \\
3,4 & $455(81 \%)$ \\
class & \\
1,2 & $336(60 \%)$ \\
3,4 & $225(40 \%)$ \\
Methotrexate use, no (\%) & $400(70 \%)$ \\
Glucocorticoid use, no (\%) & $262(47 \%)$ \\
Rheumatoid Factor, no (\%) & $287(65 \%)$ \\
anti CCP antibody, no (\%) & $137(87 \%)$ \\
\hline
\end{tabular}

Conclusion: The most common reason for discontinuation was adverse events in long term observation. The risk factors for discontinuation were class, age, and non-concomitant MTX. These results suggested that comorbidity has a significant impact on continuation rates because there are some reasons of non-concomitant MTX in addition to relate with age and the activities of daily living.

\section{REFERENCES:}

[1] Marussa B, et al. j.clin thera. 2011; 33(7): 901-913

[2] Alejandro S, et al. Rheumatol. 2016; 55(3): 523-34
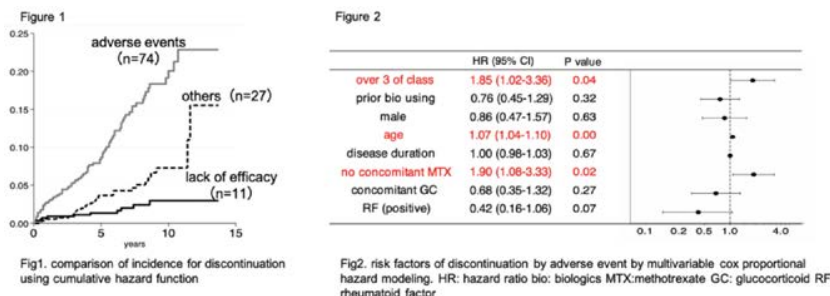

Disclosure of Interests: KENYA TERABE: None declared, Nobunori Takahashi Speakers bureau: AbbVie, Asahi Kasei, Astellas, Bristol-Myers Squibb, Chugai, Daiichi-Sankyo, Eisai, Eli Lilly, Janssen, Mitsubishi Tanabe, Ono, Pfizer, Takeda, and UCB Japan, Shuji Asai Speakers bureau: AbbVie, Astellas, Bristol-Myers Squibb, Chugai, Daiichi-Sankyo, Eisai, Janssen, Takeda, and UCB Japan, Yuji Hirano Speakers bureau: Tanabe-Mitsubishi, Pfizer, Eisai, Abbie, Chugai, Bristol-Meyers, Jansen, Astellas, UCB, Eli-Lilly, Asahikasei, Daiichi-Sankyo, Amgen, Yasuhide Kanayama: None declared, Toshihisa Kojima Speakers bureau: AbbVie, Astellas, Bristol-Myers Squibb, Chugai, Daiichi-Sankyo, Eli Lilly, Janssen, Mitsubishi Tanabe, Pfizer, and Takeda, Consultant of: AbbVie, Grant/research support from: Chugai, Eli Lilly, Astellas, Abbvie, and Novartis

DOI: 10.1136/annrheumdis-2021-eular.3387

\begin{tabular}{l|l}
\hline AB0234 & SURVIVAL OF ABATACEPT IN RHEUMATOID \\
ARTHRITIS PATIENTS: A REAL-LIFE STUDY
\end{tabular}

E. Molteni ${ }^{1}$, F. Ceccarelli ${ }^{1}$, C. Castellani ${ }^{1}$, F. Giardina ${ }^{1}$, C. Alessandri ${ }^{2}$, M. DI Franco $^{2}$, V. Riccieri ${ }^{2}$, F. R. Spinelli ${ }^{2}$, R. Scrivo ${ }^{2}$, R. Priori ${ }^{3,4}$, F. Conti $^{2} .^{1}$ Sapienza University of Rome, Rheumatology, Roma, Italy; ${ }^{2}$ Sapienza University of Rome, Dipartimento di Scienze Cliniche Internistiche, Anestesiologiche e Cardiovascolari, Roma, Italy; ${ }^{3}$ Policlinico Umberto I, Rheumatology, Roma, Italy; ${ }^{4}$ Policlinico Umberto I, Rheumatology Unit, Roma, Italy

Background: Abatacept (ABA) is a biological drug approved for the treatment of rheumatoid arthritis (RA) patients that, by working on CTLA4, can inhibit T-cell activation. Randomized controlled trials have demonstrated both the efficacy and a good safety profile, characterized by a lower infectious risk in comparison with other biological DMARDs, in RA patients. In a real-life setting, the drug retention rate could be considered as a surrogate of drug effectiveness. Data from the literature reported a retention rate of $\mathrm{ABA}$ ranging from 55 to $76 \%$ at 12 months and from 54 to $64 \%$ at 24 months (1-3).

Objectives: In the present longitudinal analysis, we evaluated the retention rate of $A B A$ in a large monocentric RA cohort.
Methods: We enrolled consecutive RA patients starting treatment with intravenous (IV) or subcutaneous (SC) ABA according to the standard of care. All the patients fulfilled the 2010 ACR/EULAR classification criteria for RA For each patient, we collected demographic parameters, serological status, previous and concomitant treatments, and disease activity by DAS28 with C reactive protein (DAS28-CRP). All the patients were assessed at baseline, and after 4 and 12 months (T4 and T12, respectively). The reasons for withdrawal of treatment were registered and classified as primary or secondary inefficacy or adverse events (AEs). Kaplan-Meier statistical analysis has been done to evaluate the survival of the treatment in patients with at least 12 months follow-up.

Results: We evaluated 161 patients [M/F 21/140; median age 67 years (IQR 21.7), median disease duration 180 months (IQR 161)]. RF was positive in $70.3 \%$ of patients, ACPA in $66.4 \%$. ABA was the first biological DMARD in 66 patients (41\%). At baseline, the median DAS28-CRP was 4.3 (IQR 1.6) and ABA was administered in association with MTX in 96 patients (59.6\%). One hundred-eleven patients $(68.9 \%$ ) started SC ABA [M/F 16/95; median age 64.5 years (IQR 21.5), median disease duration 156 months (IQR 132)], the remaining 50 IV ABA [M/F $5 / 45$, median age 71 years (IQR 60.2), median disease duration 187 months (IQR 157)]. Median age and disease duration were significantly higher in patients receiving IV in comparison with $S C A B A(p=0.008$ and $p=0.03$, respectively) We found a significant reduction of DAS28-CRP values during the follow-up in comparison with baseline [4 months: median 3.5 (IQR 1.9), $\mathrm{p}<0.0001 ; 12$ months: median 3.2 (IQR 1.4), p<0.0001]. Seven patients were lost to follow-up, in the remaining 154 patients a median treatment duration of 33 months (IQR 49) was registered. Data on drug survival are reported in Figure 1A: at 12 months, 92\% of patients persisted on treatment; this percentage decreased to $78.2 \%$ at 24 months and to $67.9 \%$ at 36 months. Furthermore, we did not find any differences in drug survival either with respect to SC vs IV administration (12 months: $93.7 \%$ versus $88.6 \%$; 24 months $78.9 \%$ versus $72.6 \%$; 36 months $63.7 \%$ versus $72.6 \%$; Figure $1 \mathrm{~B}$ ) or according to the association with MTX. Concerning the withdrawal reasons, 46 patients $(29.9 \%$ ) stopped ABA due to inefficacy (primary in 28 , secondary in 18$), 11$ patients $(7.1 \%)$ due to AEs, and 7 for inadequate adherence $(4.5 \%)$. Finally, 10 patients switched from IV to SC administration, due to patient's preference.

Conclusion: In our monocentric RA cohort, we have observed a high retention rate of ABA at both 12 and 24 months, confirming the good profile of this drug in terms of effectiveness and safety, irrespective of the route of administration and association with MTX.

REFERENCES:

[1] Cagnotto, Arthritis Res Ther 2020; (2) Salmon, J Clin Med 2020; Westhovens, Rheumatol Int 2020.

Acknowledgements: I would like to acknowledge Dr. F. Ceccarelli, for her patience.

Disclosure of Interests: None declared

DOI: 10.1136/annrheumdis-2021-eular.3689

\section{AB0235 RITUXIMAB (BIOSIMILAR) IN RHEUMATOID ARTHRITIS: CLINICAL \& IMMUNOLOGICAL RESPONSE TO VARYING DOSES- EXPERIENCES FROM A TERTIARY CARE CENTER IN SOUTH INDIA}

J. Antony ${ }^{1}$, R. Sankaralingam ${ }^{1}$, R. Maheshwari ${ }^{1}$, B. Chilukuri ${ }^{1}$, S. Chinnadurai ${ }^{1}$. ${ }^{1}$ Sriramachandra Medical College, Rheumatology, Chennai, India

Background: Rituximab (RTX) is a chimeric monoclonal antibody against CD20. There is a paucity of studies done with RTX biosimilars. This is a Retrospective and Observational study from January 2018 to December 2019 done in the Department of Clinical Immunology \& Rheumatology, Sri Ramachandra Institute of Higher Education and Research, Chennai, India.

Objectives: 1.To find the effects of varying doses of RTX in attaining clinical remission in RA.

2.To find if CD19, CD20 \& IgG help in identifying impending flare \& if these levels help in deciding the timing of the next dose of RTX.

Methods: Rheumatoid arthritis (RA) cases who were given Rituximab from January 2018 were selected. Clinical Response at 6 \& 12 months \& wherever feasible at 18 \& 24 months was assessed by Simplified Disease Activity index (SDAI). RTX initial dose was given at 0 and 14 days followed by fixed dose at six months interval.CD19, CD20 B cell count, IgG levels were tested in patients in whom it was feasible at baseline \& 6 months (select patients at 12,18 \&24 months). Patients were divided in to 5 groups (DMARD naïve, DMARD resistant \& Interstitial Lung disease (ILD) [Lung involvement $>20 \%$ in Computed Tomography $(C T)])$ and $(500 \mathrm{mg} \& 1 \mathrm{~g})$. Patients were divided into three clinical groups, (DMARD naïve, DMARD resistant \& Interstitial Lung disease (ILD) [Lung involvement>20\% in Computed Tomography (CT)]) and two treatment groups (500mg \& $1 \mathrm{~g}$ ) based on clinical indication for RTX and dose of RTX, 